\title{
EVALUATION OF ADAPTIVE PROCESSES OF KINEMATIC AND DYNAMIC PERFORMANCES OF RUNNING AT MAXIMUM SPEED IN TRAINING WITH ADDITIONAL INERTIAL LOAD APPLICATION
}

\author{
Zoran B. Pajić ${ }^{1}$
}

${ }^{1}$ Faculty of Sports and Physicial Education, Belgrade, Serbia

ORIGINAL SCIENTIFIC PAPER

DOI: 10.5550/sgia.110701.en.017P

COBISS.BH-ID: 2101528

UDC: 796.422 .015

\section{SUMMARY}

The subject of this study was to investigate the effects of additional inertial load in the training of maximum running speed. The objective of this research was to produce changes in kinematic and dynamic performances of maximum running speed in training with additional inertial loads application, as well as to determine the adaptive processes that were supposed to significantly influence the maximum running speed in the observed variables. An experiment with parallel groups was carried out during which the effect of experimental factors (inertial load) was on two levels. The first, control group (C), had no additional load. Second, experimental group (EA), ran with load attached to arms, and another group (EL), with load attached to legs. The research included initial and final measurements of variables in the phase of maximum speed $(25 \mathrm{~m}-50 \mathrm{~m})$. The change of kinematic variables recorded by non-contact telemetry measurement (two-dimensional system) of a cycle of strides during sprint step in the phase of maximum running speed was analyzed. The obtained results indicate that the applied experimental factor within the specific six week period caused statistically significant changes in experimental (EA) and (EL) groups. It is obvious that the different location of inertial load in experimental groups affected the changes in the observed variables differently, as well as that the applied additional load selectively affected the change in the observed variables. The results of this study open up new dilemmas such as the adequacy of the same load for both arms and legs, normalization of the absolute value of the load according to actual mass of each locomotive apparatus of athletes, the length of the experimental treatment, the quality of training periods in the experiment, inhomogeneity of the sample, as well as a criterion for the sample equaling according to the initial motor potential and the like.

Key Words: velocity of running, moment of inertia, moment of momentum.

\section{INTRODUCTION}

The application of external load of participants during maximum speed running has already been suggested as a potential method of training (Bosco, Rusco, \& Hirvonen, 1986; Pajić, 2000; Ropret, Kukolj, Ugarković, Matavulj, \& Jarić, 1998). Such a training of running at maximum speed with additional load should be effective in order to enable the conversion of the increased muscle strength into muscle power (Delecluse, Van Coppenolle, Willems, Leemputte, Diels, \& Gories, 1995; Ropret et al., 1998). The efficiency of muscle contraction in cases of additional load application, i.e. the ability to overcome the ad- ditional resistance by muscle contraction, depends on many factors. These are, in addition to other, higher muscle strength (based on the number of activated motor units, warming up, muscle structure, present fatigue, etc.); longer lever arm action of muscles (more convenient than a short, but specified by anatomical characteristics and cannot be changed); more convenient angle of muscle action on the lever (any moving away from the right angle by its expansion or reduction, reduces the efficiency of muscle contraction due to fewer number of contacts and myofilament surface area contacts and the like); smaller force (mass) of the additional load which opposes the muscle contraction; shorter arm 
through which the additional load force acts, a less favorable angle of action of the additional load force (further from approximately the most efficient angle of (900), thus reducing the efficiency of the additional load resistant force and facilitates muscle to overcome, i.e. control the additional load force). Previous statements can be condensed into the general rule that everything that acts on the increase of the muscle contraction torque force and/ or the reduction of resistance forces torque contributes to the muscle contraction efficiency, i.e. the ability to overcome the additional load force (resistance) with smaller/ lower strength. Different results have been obtained for the fact that the kinematic and dynamic variables of running at maximum speed change under the influence of additional load depending on its weight and location of its fixing, and they are probably the consequence of different methodologies applied in researches. The experiments that were carried out have implied the use of various load size, places of their fixing and running at different speeds. However, measurements while running at a moderate speed and on the treadmill were conducted in most studies, with the exception of some in which the change of kinematic variables in conditions of maximum speed and acceleration with the additional load was analyzed (Majdell \& Alexander, 1991; Ropret et al., 1998). In some studies the results showed no change in stride length and frequency (Catlin \& Dressendorfen, 1979; Cavanagh \& Kram, 1989; Martin \& Cavanagh, 1990). The results indicating increased frequency and reduced stride length were obtained in most papers (Cooke, McDonaght, Nevill, \& Davies, 1991; Rusko \& Bosco, 1987; etc.). The opposite results, when the frequency was reduced and stride length increased, have been reported in the researches (Martin, 1985; Stegman, 1981; Winter, 1983; etc.). The result when the increased arm load and leg load in particular caused a reduction in running speed, while the stride length remained unchanged and frequency reduced, was obtained (Ropret et al., 1998). Majdell and Alexander (1991) verified the change of the kinematics of $40 \mathrm{~m}$ running at a maximum speed, under the influence of training with the additional load. Six-week training with the additional load (10 pounds, $4.5 \mathrm{~kg}$ ) was applied in the experiment. The treatment influenced the maximum speed of running to be statistically significantly increased (from $5.16 \mathrm{~m} / \mathrm{s}^{-1}$ to $5: 26$ ), to shorten the contact phase (from 0.13 to $0.11 \mathrm{sec}$ ), to statistically significantly reduce the upper leg angle of the take off leg in a moment of take-off (from 4.27 to 4.12 degrees), as well as and to increase the maximum angle of the lower leg flexion (from 2.62 to 3.28 degrees). The variables stride frequency and stride length significantly correlate with the morphological characteristics, and the additional load affects the decline of their relationship with morphological characteristics (Pajić, Preljević, \& Kostovski, 2010). It is not completely clear whether the experimental factor caused the loss of correlation relationships, or the reason for that was inhomogeneity of the sample (morphological and/or motor mismatch) or both.

The objective of this research was to analyze the effects of additional inertial load in the training process on the maximum running speed. The applied experimental factor should cause changes in kinematic and dynamic performances of maximum running speed in order to determine the adaptive processes in the observed variables. Such a training of running at maximum speed with the additional load should be effective to facilitate the conversion of increased muscle strength into muscle power. (Delecluse et al., 1995; Ropret et al., 1998).

Thus, the goal of this study was, after their identification, to evaluate the adaptive processes that result from training with additional inertial load application in kinematic and dynamic performances of maximum speed running, which is assumed to significantly affect the maximum running speed.

\section{METHODS}

\section{Sample of participants}

The sample of participants in this study consisted of students of Faculty of Sport and Physical Education in Belgrade. From the current popula-tion a sample of students was defined $(n=21)$, aged 20.8 \pm 1.8 . Due to the initial sprint time the participants were classified into three groups depending on the running speed. The groups were formed in attempt to make on equal abilities distribution among them.

\section{Measurement conditions and variables (Instruments)}

The system of three pairs of solar cells was applied for time parameters measurement - measurement system (Brower timing system).

The following variables were measured:

\begin{tabular}{lcc}
\hline \multicolumn{1}{c}{ Variables } & Sign & $\begin{array}{c}\text { Meas. } \\
\text { Unit }\end{array}$ \\
\hline $\begin{array}{l}\text { Running speed in the } \\
\text { maximum speed phase }\end{array}$ & VR & $\mathrm{m} / \mathrm{s}$ \\
\hline $\begin{array}{l}\text { The average stride length } \\
\text { in the maximum speed phase }\end{array}$ & $\mathrm{SL}$ & $\mathrm{m}$ \\
\hline $\begin{array}{l}\text { Stride frequency in } \\
\text { the maximum speed phase }\end{array}$ & $\mathrm{SF}$ & $\mathrm{Hz}$ \\
\hline
\end{tabular}


For the number of strides (NS) measurement, the system of three video cameras was used, one of which recorded the distance of $25-50 \mathrm{~m}$ in order to determine the number of strides between the first and the last contact, while the first and the last contact were identified by the other two cameras. The average stride length (SL) was calculated by dividing the distance between the first and the last foot contact with a number of strides. Stride frequency (SF) was calculated by dividing the speed of running with the length of strides. Based on the time for which the distance was run, the maximum speed of running was calculated (VR). For kinematic parameters measurement Mac Reflex 3.2 Measurement System Software with the Macintosh version 3.0 (for $2 \mathrm{D}$ and $3 \mathrm{D}$ system) was applied. The measurement procedure with this system provides a non-contact (telemetric) movement measurement. The basic principle of the system is recording the position of a number of well-defined points in space.
The measured points are marked with reflective markers, and their movements are registered by a special video camera.

\begin{tabular}{lcc}
\hline \multicolumn{1}{c}{ Variables } & Sign & $\begin{array}{c}\text { Meas. } \\
\text { Unit }\end{array}$ \\
\hline $\begin{array}{l}\text { Angle in the knee joint } \\
\text { centre at take-off time }\end{array}$ & AKTT & $\circ$ \\
\hline $\begin{array}{l}\text { Angle in the knee joint } \\
\text { centre at the time of contact }\end{array}$ & AKTC & $\circ$ \\
\hline $\begin{array}{l}\text { Angle of maximum } \\
\text { lexion of the lower } \\
\text { leg during the back swing }\end{array}$ & AMFL & $\circ$ \\
\hline $\begin{array}{l}\text { Angle of maximum } \\
\text { llexion of the forearm } \\
\text { during the back swing }\end{array}$ & AMFA & $\circ$ \\
\hline
\end{tabular}

The kinematic variables measured on the track in conditions of maximum running speed (Figure 1):

FIGURE 1

Kinematick variables

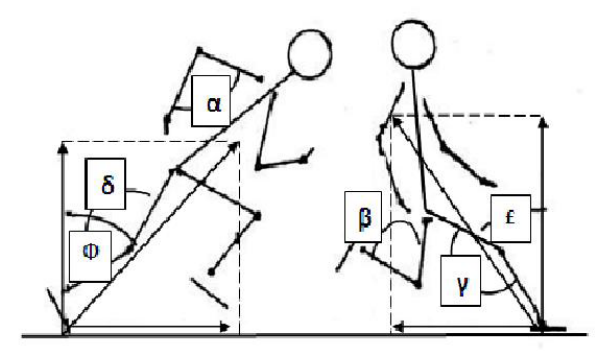

Legend: $(\boldsymbol{\delta})$ - angle in the knee joint center at take-off time (AKTT); $(\alpha)$ - angle of maximum flexion of the forearm during the back swing (AMFA); $(\boldsymbol{\beta})$ angle of maximum flexion of the lower leg during the back swing (AMFL); $(\gamma)$ - angle in the knee joint center at the time of contact (AKTC); $(\boldsymbol{\Phi})$ - critical angle of the body at take-off time; $(\mathfrak{E})$ critical angle of the body at the time of contact.

Based on the previous measurements and software data acquisition with the applied software Mac Reflex 3D.V.3.1B2 and Microsoft Excel software adaptable, the values for variables (AKTT, AMFL, AMFA and AKTC) were determined.

\section{The experiment protocol}

The experiment with parallel groups was carried out during which the effect of the experimental factor (inertial load) was on two levels. The first control group $(\mathrm{C})$ realized the sprint training without additional load application. The second experimental group (EA) ran with the additional load attached to the arms. The third experimental group (EL) ran with the additional load attached to the legs.

\section{Experimental factor}

In order to increase the moment of the arms and legs inertia in the training procedure the addi-tional load was applied in the form of bands with plates, fixed to the ankle and wrist. In line with previous researches and results (Catlin \& Dressendorfen, 1979; Cavanagh \& Kram, 1989; Martin, 1985; Ropret et al., 1998; Stegemann, 1981) a load of $1.8 \mathrm{~kg}$ was applied, for which it was calculated that it changed the average torque inertia for about $50 \%$. 


\section{Testing procedure}

The research included initial and final measurements of all variables. Both measurements were realized in two days, the initial measurement (pretest) one day prior to the beginning of training procedures application, and the final one two days after the end of the training procedure. The measurement of dynamic and kinematic variables was realized in running at maximum speed on the track from the 25th to 50th meter. Each participant ran two times, and for the final processing, the better result was used. Measuring devices (photocells - Brower Timing System) were set at the start, to register the stand position start $(0.5 \mathrm{~m})$, at $25 \mathrm{~m}$ and at the end of $50 \mathrm{~m}$. The values were measured with an accuracy of $.01 \mathrm{~s}$.

\section{Training procedures}

Every athlete warmed up before the active speed training. Warm-up consisted of a number of sprint exercise (dog run, high lift your knees, scooping up, skip-semi-skip running etc.), and stretching all the muscles involved in sprinting. They then performed a series of acceleration and change of pace running. After this warm-up the respondents were marshaled into their respective groups to carry out specific procedures for trainers. During the six week training the exercise was performed three times a week; it was progressively more difficult, because the volume of work increased after every two weeks. In the first two weeks, each subject was performing a series of five repetitions of his specific training regime. During the third and fourth week of training the load is increased to two sets of five repetitions of their specific training. Last two weeks, the intensity was increased to three sets of five repetitions for each group. One series consisted of five repetitions, and ran a top speed of 50 meters from semi-high start with 2-3 minutes rest between each run, with 8-10 minutes recovery between each set.

\section{Training method}

- First, the control group (C) included a run at maximum speed at $50 \mathrm{~m}$, from semi-high start, with a $2-3 \mathrm{~min}$. recovery between reps and 8-10 min. rest between sets.

- Second, the experimental group (EA), included the same training program that the control group implements, with the provision that the two hands bore the additional load.

- Third, the experimental group (EL), included the same training program that the control group implements, with both feet carrying an additional burden.

\section{Acquisition of experimental results}

Out of the descriptive statistical indicators, the measures of central tendency (arithmetic mean $-\mathrm{M}$ ) as well as measures of dispersion (standard deviation - SD) were applied. Out of the qualitative methods of statistical analysis, the $t$-test for dependent samples was applied. The level of significance $(\phi<.05)$ was applied to determine the significance of differences between pretest and post test for each group. Statistical analysis was performed in the statistical program SPSS 16.

\section{RESULTS AND DISCUSION}

The results of the descriptive statistics for all three groups at the initial and the final measurement are displayed in Table 1.

The results of $t$-test for dependent samples at the initial and the final measurement are shown in the Table 2.

From the shown results it can be concluded that the applied training procedure in the maximum speed phase did not cause significant changes in the running speed (RS) in either group. The comparison of results from the initial and final measurements showed that there were no statistically significant changes in the observed variables in the control group (C).

From the above it is evident that the participants of the group (EL) reduced the running speed, but not within the range of statistical significance. Similar to these findings, (Ropret et al., 1998) stated that the effect of leg load on speed was statistically significant in both sprint stages (speed decline of $7.8 \%$ and $12.8 \%$ ). The reasons for speed decline in the experimental group (EL) might be found in the assumption that the applied load caused a technique and movement coordination disruption respectively, i.e. an inadequate adaptation of the nervous system in movement control. Another reason could be a deficit in the speed (reactive) force that is needed in the new modified conditions of overcoming the gravitational forces, ground reaction force and the lower extremities inertia. Shortening of the contact phase, one of the key factors in increasing the running speed is largely the ability to express force for the shortest possible time $(F-t)$, greater exhibited force and displacement to the right $(F-v)$ relationship. If in such a study a significant shift $(F-v)$ curve to the right was determined, it would clearly demonstrate that exercise with additional load modified the mechanical work of leg muscles in ex- 
perimental group athletes, i.e. it increased the speed (reactive) power. In the experimental groups there are statistically significant differences in variables for the average stride length (SL) as follows - on average the participants had a lower score on the initial measurement $(\mathrm{EA}-p<.022)$ and $(\mathrm{EL}-p<.003)$ and the stride frequency variable $(\mathrm{SF})$ - the participants on average had higher scores on the initial measurement $(\mathrm{EA}-p<.002)$ and $(\mathrm{EL}-p<.000)$. Therefore, there was an extension of strides and the reduction of their frequency.

\section{TABLE 1}

Descriptive statistics at the initial and the final measurement for the control group (C), experimental (EA) and experimental (EL) groups.

\begin{tabular}{llrrrrrr}
\hline \multirow{2}{*}{ Variables } & $\begin{array}{c}\text { Measure- } \\
\text { ment }\end{array}$ & \multicolumn{1}{c}{ Control (C) } & \multicolumn{2}{c}{ Experimental (EA) } & \multicolumn{2}{c}{ Experimental (EL) } \\
\cline { 2 - 9 } VR & final & 8.11 & .45 & 8.45 & .27 & 8.29 & .30 \\
\cline { 2 - 9 } & initial & 8.10 & .35 & 8.45 & .35 & 8.33 & .37 \\
\hline \multirow{2}{*}{ SL } & final & 2.01 & .12 & 2.16 & .14 & 1.98 & .23 \\
\cline { 2 - 9 } & initial & 1.96 & .20 & 2.08 & .14 & 1.88 & .24 \\
\hline \multirow{2}{*}{ SF } & final & 4.02 & .20 & 3.92 & .24 & 4.22 & .46 \\
\cline { 2 - 9 } & initial & 4.19 & .27 & 4.07 & .29 & 4.47 & .47 \\
\hline \multirow{2}{*}{ AKTT } & final & 160.83 & 4.66 & 166.16 & 1.16 & 161.00 & 1.41 \\
\cline { 2 - 8 } & initial & 160.66 & 4.63 & 160.33 & 1.03 & 162.66 & 1.03 \\
\hline \multirow{2}{*}{ AKTC } & final & 158.33 & 3.50 & 164.00 & 2.09 & 155.33 & 1.75 \\
\cline { 2 - 8 } & initial & 156.83 & 4.87 & 157.00 & 2.28 & 159.00 & 1.09 \\
\hline \multirow{2}{*}{ AMFL } & final & 38.83 & 2.85 & 43.00 & 3.63 & 45.66 & 3.72 \\
\cline { 2 - 8 } & initial & 39.66 & 2.33 & 42.16 & 4.87 & 42.50 & 4.08 \\
\hline \multirow{2}{*}{ AMFA } & final & 104.83 & 3.48 & 97.50 & 3.78 & 113.16 & 4.21 \\
\cline { 2 - 8 } & initial & 106.33 & 3.72 & 106.33 & 4.50 & 106.00 & 6.09 \\
\hline \multirow{2}{*}{ V } & & & & & & &
\end{tabular}

Legend: VR - running speed in the maximum speed phase; SL - the average stride length in the maximum speed phase; $\mathbf{S F}$ - stride frequency in the maximum speed phase; AKTT - angle in the knee joint center at take-off time; AMFA - angle of maximum flexion of the forearm during the back swing; AMFL - angle of maximum flexion of the lower leg during the back swing; AKTC - angle in the knee joint center at the time of contact.

When wearing the additional load, the moment of inertia is increased, resulting in a lower frequency of the leg which acts as a pendulum (Stegman, 1981). This increases swing length phase, while the reduced speed of leg during swing can be a limiting factor to a further running speed increase (Bobbert, Mackay, Schinkelshoek, \& Huijing, 1986; Martin, 1985). Thus, under the assumption that motion speed is unchanged, stride frequency reduction is followed by stride length increase. In accordance with the results of this study on stride length, there were also findings in which the increase in stride length was observed when the effect of adding $2 \mathrm{~kg}$ on each foot during walking was taken into account (Inman, Ralston, \& Todd, 1981). Russsel and Belding (1964) reported that the stride frequency is inversely proportional to the weight of footwear worn during walking at speed of 3.5 miles $/ \mathrm{h}$. It is evident that the arms load caused smaller decrease in stride frequency rather than the legs load, although the decline in stride frequency was statistically significant in both groups. The same results that the stride frequency decreased less in the group (EA) in both phases (1.6\% and $1.5 \%$ ) were reported by Ropret et al. (1998). The explanation for this could be the fact that the arm adjustments to the changed conditions of moment of inertia, and therefore the gravitational forces, are much bigher compared to the legs. They happen because of the tendency to maintain the movement intensity, so there is a reduction in the upper armforearm angle, to eliminate the forces acting on the longer arm, i.e. torque reduction, which causes the frequency increase. The increased arm frequency opposed to legs cause the increased level of neural activity (reciprocal inhibition) and thus positively influences the leg kinematics. Bearing in mind that the amplitude reduction of the swing is more possible in 
the elbow than in the knee and hip joint (Majdell \& Alexander, 1991), it can be assumed that such adaptive processes, as a result of the arm load, may influence the change in leg kinematics. These claims can be linked to the findings (Cook et al., 1991) that the additional load of $5 \%$ of the total body mass, worn as a belt on the trunk, is much higher than in this study, caused an increase stride frequency. It may be concluded that the higher load caused an increased nerve pulsing to the periphery, in order to maintain the movement intensity, resulted in stride reduction, thereby increasing the stride frequency. The adaptation of the body in mentioned circumstances is of neurogenic and myogenic nature (Bosco, Zanon, Rusko, Dalmonte, Bellotti, Latteri et al., 1984; Milner-Brown et al., 1975; Moritan \& De Vries, 1979; Thornton \& Rummel, 1974; ac-

TABLE 2

Descriptive statistics at the initial and the final measurement for the control group $(C)$, experimental (EA) and experimental (EL) groups.

\begin{tabular}{|c|c|c|c|c|c|c|c|c|c|}
\hline \multirow[b]{2}{*}{ Variables } & \multicolumn{3}{|c|}{ Control (C) } & \multicolumn{3}{|c|}{ Experimental (EA) } & \multicolumn{3}{|c|}{ Experimental (EL) } \\
\hline & $t$ & $d f$ & $\begin{array}{c}\text { Sig. } \\
\text { (2-tailed) }\end{array}$ & $t$ & $d f$ & $\begin{array}{c}\text { Sig. } \\
\text { (2-tailed) }\end{array}$ & $t$ & $d f$ & $\begin{array}{c}\text { Sig. } \\
\text { (2-tailed) }\end{array}$ \\
\hline VR & -.874 & 5 & .422 & -.015 & 5 & .988 & -.626 & 5 & .559 \\
\hline SL & 1.270 & 5 & .260 & 3.272 & 5 & .022 & 5.423 & 5 & .003 \\
\hline SF & -1.572 & 5 & .177 & -5.890 & 5 & .002 & -7.998 & 5 & .000 \\
\hline AKT'T & .415 & 5 & .695 & 10.750 & 5 & .000 & -2.712 & 5 & .042 \\
\hline AKTC & 1.307 & 5 & .248 & 8.573 & 5 & .000 & -8.696 & 5 & .000 \\
\hline AMFL & -1.746 & 5 & .141 & 1.185 & 5 & .289 & 10.304 & 5 & .000 \\
\hline AMFA & -2.423 & 5 & .060 & -22.007 & 5 & .000 & 3.909 & 5 & .011 \\
\hline
\end{tabular}

Legend: VR - running speed in the maximum speed phase; SL - the average stride length in the maximum speed phase; $\mathbf{S F}$ - stride frequency in the maximum speed phase; AKTT - angle in the knee joint center at take-off time; AMFA - angle of maximum flexion of the forearm during the back swing; AMFL - angle of maximum flexion of the lower leg during the back swing; AKTC - angle in the knee joint center at the time of contact.

The results of this study indicate that the experimental factor significantly influenced the changes in elbow and knee joint angles while realizing the maximum running speed, and thus its efficiency, given the importance of these variables for success at maximum speed running. It is obvious that the different location of inertial load in experimental groups affected the changes in the observed variables differently, as well as that the applied additional load selectively affected the change in the observed variables. The applied training procedures in the phase of maximum speed in the experimental groups (EA) and (EL) caused a statistically significant change of the knee joint centre angle at the time of take-off cording to Bosco et al., 1986; ${ }^{1}$ Russo \& Bosco, 1987) and it is carried out by a relatively quick adaptation of neural factors by increasing the number of recruited motor units, the increased level of discharge and their better mutual work synchronization. However, the second phase (of myogenic adaptation), is characterized by the increase of their glycolytic potential and lasts for several months (McDonagh \& Davies, 1984; according to Bosco et al., 1984), which justly points to the need of a special study of experimental treatment duration and possibly leads to doubt regarding the length of the treatment applied in this study given the different plasticity of some tested variables.

${ }^{1}$ First three papers were cited in the article Bosco et al, 1986 , and that is why they are not in the list of references 
sulted in a more positive impact of horizontal component of the ground reaction force on the speed of running in the ventral direction.

The applied training procedure of the experimental groups (EA) and (EL) caused a statistically significant change in the variable the centre of the knee joint angle at the time of contact (AKTC) - the participants on average had a lower score in (EA) $p<.000$ ) and higher score in the initial (EL) $p<.000)$. The results indicate that the increase in angle in (EA) had a positive impact, while the reduction in (EL) negatively affected the running speed. So, there was an increase in knee flexion, which negatively affects the maximum running speed. In order to achieve a higher running speed at the time of contact with the ground a lower critical angle of the body on contact, or greater angle in the knee during contact (AKTC) are required i.e. lower flection, because it reduces the negative impact of the horizontal component of ground force reaction on the running speed in dorsal direction.

From the aforementioned results it is noted that the participants in the experimental group (EL) increased the average angle of maximum flexion of the lower leg during the last swing (AMFL) within the statistical significance - the participants on average had a lower score on the initial $(\mathrm{EL}-p<.000)$. This change has a negative effect on running speed since the moment of leg inertia is increased; the angular velocity and thus leg frequency are both reduced. The maximum forearm angle flexion during the back swing (AMFA) changed significantly - the participants on average had higher scores in the initial measurement $(\mathrm{EA}-p<.000)$ and lower scores $(\mathrm{EL}-p<.011)$. The monitoring of arm kinematics during running with the additional load would make it possible to prove the assumption that the above changes are the result of adjustments in the mechanics of arm movements, since the participants performed the elbow joint flexion by which the moment of inertia is reduced, and thus the overall impact of the additional load. Since the smaller angle reduces the hand inertia moment, therefore its higher speed and shorter duration of arm swing are achieved.

This results in frequency increase of the body segments movements, i.e. the positive influence of the higher running speed. The explanation for this could be the fact that the arm adjustment to the changing conditions of moment of inertia is much higher compared to legs. They happen, as it has already been explained, because of the tendency to maintain the intensity of movement, which leads to reduction of the upper arm-forearm angle, in order to eliminate the forces acting on the longer arm, i.e. to reduce the torque, which causes an increase in frequency.

For the evaluation of the obtained results of (AMFL and AMFA) variables the following facts may serve. Human movements are not made by individual segments, but by kinetic chains that comprise larger number of joints, among which there has to be good coordination. It helps developing a force against the external load, the accuracy of the movement, which is especially important; it ensures that the ends of the kinetic chains develop much faster than those achieved by a single segment. This movement coordination results in more speed concordance of adjacent joints of the kinetic chain in the same direction for high speed of a certain segment of kinetic chain. When running at maximum speed, the arm speed is the result of the sum of upper arm, forearm and the hand speeds. Thus, as the result of time coordination achieved in the joints of long halfkinetic chains, the maximum speed of skeletal muscles shortening (which is less than $1 \mathrm{~m} / \mathrm{s}$ ), transforms into a higher distal segment speed (hand speed during swing is about $15 \mathrm{~m} / \mathrm{s}$, and foot speed of the swing leg is over $20 \mathrm{~m} / \mathrm{s}$ ). At sprint running, it is the chain of the foot resting on the ground, through the pelvis, to the feet of swing leg, while in the second case from the same foot, over the trunk and arms, to the hand. At these activities the open successive schemes are realized because the movement is transferred from a closed to an open end of the kinetic chain. A sprinter cannot change the mass of his kinetic chains and segments, but can affect the momentum of quantity of his motion (the product of moment of inertia and angular velocity changes). This is achieved in a way that by the rotational motion in the shoulder, hip and ankle the change of moment of inertia of his kinetic chains happens. So, at the same angular momentum, by reducing the moment of inertia of the kinetic chain, or of an active body segment, its angular velocity can be increased or by increasing the moment of these segments inertia the radial velocity can be reduced. So, by changing the moment of segments inertia, a sprinter controls its angular velocity. A leg and/or an arm rotate around the frontal axis in the swing phase, which passes through the centre of the hip or shoulder. In order to make this movement for the shortest possible time the respondents acted with the maximum moment of force of the current muscles. Since this moment of muscle action is limited, the respondent reduces moment of segments inertia. By knee (AMFL) or elbow flexion (AMFA) he approaches the centres of gravity of the lower leg and foot to the axis of rotation, i.e. hip joint, or the centres of gravity of forearms and 
hands to shoulder joint. Thus the moment of inertia of the kinetic chains is reduced by about two times, according to Newton's second law (the total moment of force acting on a body fixed to the axis around which can rotate, is equal to the product of its moment of inertia and its angular acceleration), angular acceleration of the swing leg or arm is increased for the same amount. By this their higher speed and shorter duration of the swing phase are achieved. This results in increasing the frequency of the body segments movements and higher running speed.

It is interesting that, despite the foregoing, the experimental group (EL) significantly increased the maximum knee flexion angle (AMFL). It could be expected that the respondents would adapt to the changed inertia conditions and make adequate knee flexion and reduce the angle. But this did not happen. According to Newton's first law, objects stays in motion with the same speed and in the same direction unless acted upon by an unbalanced force. Every body resists the action of an external force by its own inertia proportionally to its own weight (body which changes its motion under the force of another body, due to inertia affects another body by force of inertia). Thus, the swing leg exerts a force of inertia to other segments of the body in the opposite direction of its motion (acceleration). Hence, this force is directly dependent on the mass segment and the size of its acceleration. In this experiment, the segment mass is increased by the additional load, which caused the increase of the inertia forces with all the subsequent consequences. Inertia forces in sprint running during the continuous leg swings act constantly, causing a larger quantity of muscle work to be spent on overcoming this force than all the others (work against gravitational force, air resistance, ground friction force, and the like). So, the reason for the increase in the maximum knee flexion angle (AMFL) could be the assumption that for the newly altered inertial conditions there were no adequate changes in the power and strength or that training period in the experiment did not provide enough time for proper motor adaptation in the mentioned variables of force and power. It is possible that the reason for an inadequate adaptation may be sample motor inhomogeneity (in the power and strength), given that the sample is balanced solely by the results in sprint at the required distance. Thus, perhaps, excessive heterogeneity of individual results affected negatively the overall variance (the dispersion of results). It is possible that, by adding the above factors, the conditions which determine the speed decrease in this experimental group were created.
However, an especially interesting finding is that in the group (EL) there was a significant increase in the elbow joint angle (AMFA) even though the hand was not additionally loaded. It could be expected that the respondents would adapt to the changed inertia conditions and make adequate elbow joint flexion and reduce the angle and the moment of inertia. This finding could be evaluated by the following facts. According to Steiner's theorem $\left(\mathrm{mr}^{2}\right)$, the moment of inertia of the current segment or any of the kinetic chains, equals the sum of its own (equal to the moment of inertia which a segment would have if the axis of rotation moved into its centre of gravity) and positional (equal to the product of mass segments or the whole kinetic chain and the square of the distance of its centre of gravity from the axis of rotation) moment of inertia. The more the segment is moved away from the axis of its rotation, the greater its moment of inertia is. This means that during moving the segment from the axis of rotation, its own moment of inertia does not change, but the positional increases by the square of this distance. Since there was a statistically significant increase in knee angle (AMFL), there was an increase in positional moment i.e. the increase of force of inertia of a given segment. The swing leg exerts a force of inertia to other segments of the body in the opposite direction of its acceleration. Thus, when running at maximum speed, there is a constant acceleration and deceleration of segments (kinetic chain of limbs), especially of their open ends - hands and feet, during which great forces of inertia of the additional loads must be compensated by muscle forces. Synchronized movement of contralateral limbs is one of the main characteristics of fundamental motions, and the ipsilateral limbs are always in the opposite movement phase. Therefore the opposite movement is always done in the hip joint and shoulder of the same side of the body in the sagittal plane (ventrally - dorsal). This synchronization of movements of the upper and lower limbs affects the hip to follow the movements of legs in ventral and dorsal direction (leading to pelvis rotation in the transverse plane), and makes the shoulder joint follow the movement of arms. The consequence of this is that the pelvis and shoulder axis in the transverse plane always rotate in opposite directions. Since, in the ventral direction, the hip of the leg in the swing phase and the shoulder joint of contralateral hand move at the same time, it implies that the rotation of the spine is always in the direction of the swing leg. This could lead to the conclusion that the increase in positional moment of arm inertia is an attempt to compensate for the increased inertia forces of a leg moving in the opposite direction. There- 
fore it could affect a better synchronization of contralateral segments' movements, with the purpose of more stable overall movement of the lever system of the entire body.

\section{CONCLUSION}

The obtained results indicate that the applied experimental factor within the specific six week period caused statistically significant changes in experimental (EA) and (EL) groups. The obtained results indicate that the applied experimental factor in the specific six week period caused statistically significant changes in experimental (EA) and (EL) groups. The applied load significantly influenced the kinematic variables which influence on the factors that favour and those that interfere with the efficiency of performance of maximal running speed is obvious (angular momentum, moment of inertia, ground reaction forces, gravitational forces, monoarticular and biarticular muscle action, matching movements, activities of the kinetic chain). We can assume that the variables (AKTT, AKTC, AMFL and AMFA) are predictable for the maximum running speed and that there is their significant connection with running at maximum speed.

The results of this study face a new dilemma. It is not clear whether the same load for arms and legs is adequate, since the arm adjustment to the changed conditions of moment of inertia and therefore the gravitational forces is much higher compared to the leg adjustment, as well as whether different time is needed for full adaptation of both arms and legs. In this research an attempt to increase the momentum by changing the inertia conditions is significant. The introduction of the absolute value of the load, without calculating the actual mass of each locomotive apparatus individually, raises doubts because the moment of inertia increases by $\mathrm{mr}^{2}$, i.e. the value of the applied load is squared, even if it is only one gram. The results of this study reasonably indicate the need for special study of experimental treatment duration and eventually doubt the length of the applied treatment in this study, given the different plasticity of some tested variables. So, there were no adequate changes in the power and strength or the training period in the experiment did not provide enough time for proper motor adaptation in the mentioned variables of force and power. It is possible that the reason for an inadequate adaptation can be motor (in the power and strength) sample inhomogeneity, given that the sample is balanced solely by the results in a sprint at the required distance. It can be assumed that, to- gether with the prolonged duration of training factor influence, there would be a correction in speed power expression, and consequently an easier correction of altered inertial conditions for loaded body segments, and thus the relative increase of movement frequency. This would probably increase the running speed. Given the finding that it is not clear whether the experimental factor caused the loss of correlation links between the dynamic variables and morphological characteristics (Pajić et al., 2010), or the reason for it is inhomogeneity of the sample (morphological and/or motor mismatch) or both, it may be interesting to examine the predictability and the impact of morphological characteristics in training with the additional load application.

\section{REFERENCES}

Bobbert, M. Y., Mackay, M., Schinkelshoek, D., \& Huiijing, P. A. (1986). Biomechanical analysis of drop and countermovement jumps. Eur. J. Appl. Physiol., 54, 566-573.

Bosco, C., Zanon, S., Rusko, H., Dalmonte, A., Bellotti, P., Latteri, F., et al (1984). The influence of extra load on mechanical behavior of skeletal muscle. Eur. J. Appl. Physiol., 53, 149-154.

Bosco, C., Rusko, H., \& Hirvonen, J. (1986). The effects of extra - load conditioning on muscle performance in athletes. Med. Sci. Sports Exerc., 18(4), 415-419.

Catlin, M. J., \& Dressendrfer, R. H. (1979). Effects of shoe weight on the energy of running. Med. Sci. Sports, 11, 80.

Cavanagh, P. R., \& Kram, R. (1989). Stride length in distance running: velocity, body dimensons, and added mass effects. Med. Sci. Sport Exerc, 21(4), 467-479.

Cooke, C. B., McDonagh, M. J. N., Nevill, A. M., \& Davies, T. C. M. (1991). Effects of load on oxygen intake in trained boys and men during treadmill running. J. Appl. Physiol., 71(4), 1237-1244.

Delecluse, C., Van Coppenolle, H., Willems, E., Leemputte, M., Diels, R., \& Goris, M. (1995). Influence of high-resistance and high-velocity training on sprint performance. Med Sci Sport Exerc, 27, 1203-1209.

Inman, V. T., Ralston, H. J., \& Todd, B. (1981). Human walking. Baltimore: Williams and Wilkins,

Majdell, R., \& Alexander, M. J. (1991). The effect of overspeed training on kinematic variables in sprinting. J. Human Mov. Studies., 21, 19-39. 
Martin, P. E. (1985). Mechanical and physiological responses to lower extremity loading during running. Med. Sci. Sports Exerc., 17(4), 427-433.

Martin, P. E., \& Cavanagh, P. R. (1990). Segmental interactions within the swing leg during unlosded and loaded running. J. Biomechanics, 23(6), $529-536$.

Pajić, Z. (2000). The influence on inertial load on adaption processes during running at various speeds. Fizička kultura, 54(1/4), 46-55.

Pajić, Z., Preljević, A., \& Kostovski, Z. (2010). Effects of the application of inertial loads on the morphological performances in the stage of running development at maximaum speed. International Scientific Journal of Kinesiology, 7(2), 47-53.

Ropret, R., Kukolj, M., Ugarković, D., Matavulj, D., \& Jarić, S. (1998). Effects of arm and leg loa- ding on sprint performance. Eur J Appl Physiol., 77, 547-550.

Rusko, H., \& Bosco, C. (1987). Metabolic response of endurance athletes to training with added load. Eur. J. Appl. Physiol., 56, 412-418.

Russell, H. D., \& Belding, H. S. (1964). Metabolic cost of wearing various types of footwear. National Academy of Sciences Committee on Quartermaster Problems Report. Cambridge, MA; Harward Fatique Laboratory.

Stegman, J. (1981). Exercise physiology: Physical bases of work and sport. J.S. Skinner (Trans. and Ed.). Chicago: Year book medical publishers.

Winter, D. A. (1983). Biomechanical motor patterns in normal walking. J. Mot. Behav., 15, 302-330.

Received: February 6, 2011

Accepted: April 26, 2011

Correspondence to: Zoran Pajić, PhD

Faculty of Sports and Physical Education

Blagoja Parovića 156 11030 Belgrade Serbia

Phone: 0038163234915

E-mail: zoran.pajic@dif.bg.ac.rs 